\title{
Spatial distribution of tuberculosis in a rural region of Western Province, Papua New Guinea
}

\author{
Tanya Diefenbach-Elstob, a,b Vanina Guernier-Cambert, ${ }^{b}$ Bisato Gula, ${ }^{c}$ Robert Dowi, ${ }^{c}$ Daniel Pelowa, ${ }^{c}$ William Pomat, ${ }^{d}$ \\ Catherine Rush,, $\mathrm{a} b$ David Plummer, ${ }^{a}$ Emma McBryde, ${ }^{b}$ and Jeffrey Warner, ${ }^{a, b}$ \\ Correspondence to Tanya Diefenbach-Elstob (email: tanya.diefenbachelstob@gmail.com)
}

Introduction: There is a high burden of tuberculosis (TB) in the Western Province, Papua New Guinea. This study aims to describe the spatial distribution of TB in the Balimo District Hospital (BDH) catchment area to identify TB patient clusters and factors associated with high rates of TB.

Methods: Information about TB patients was obtained from the BDH TB patient register for the period 26 April 2013 to 25 February 2017. The locations of TB patients were mapped, and the spatial scan statistic was used to identify high- and low-rate TB clusters in the BDH catchment area.

Results: A total of 1568 patients were mapped with most being from the Balimo Urban $(n=252)$, Gogodala Rural ( $n=1010$ ) and Bamu Rural $(n=295)$ local level government (LLG) areas. In the Gogodala region (Balimo Urban and Gogodala Rural LLGs), high-rate clusters occurred closer to the town of Balimo, while low-rate clusters were located in more remote regions. In addition, closer proximity to Balimo was a predictor of high-rate clustering.

Discussion: There is heterogeneity in the distribution of TB in the Balimo region. Active case-finding activities indicated potential underdiagnosis of TB and the possibility of associated missed diagnoses of TB. The large BDH catchment area emphasizes the importance of the hospital in managing TB in this rural region.

W estern Province in Papua New Guinea (PNG) has a very high burden of tuberculosis (TB) with a case notification rate of 674 per 100000 people in 2016. ${ }^{1} \mathrm{~TB}$ is known to cause a high burden of disease in Balimo and the Gogodala region of the Middle Fly District; the average reported incidence of TB at Balimo District Hospital (BDH) was 727 cases per 100000 people per year from 2014 to 2016 for people in the combined Balimo and Gogodala local level government (LLG) areas. $^{2}$ Furthermore, rates of paediatric and extrapulmonary TB have been identified as very high, with $25.0 \%$ of patients aged $0-14$ years, and $77.1 \%$ of patients diagnosed with extrapulmonary TB infection. ${ }^{2}$

$\mathrm{BDH}$ is the primary facility providing TB diagnosis and DOTS-based treatment in the Gogodala region. Other smaller health facilities, including health clinics and aid posts, can provide limited TB services such as clinical extrapulmonary TB diagnosis and treatment and pulmonary TB services when a sputum sample is not able to be transferred to the town of Balimo. ${ }^{2}$ Given the high burden of $\mathrm{TB}$ reported at $\mathrm{BDH}$, there is a need to understand the distribution of TB in the Balimo region. Such analysis will provide insight into areas with high and low rates of TB as well as evidence to support the focused delivery of TB services. This study used spatial epidemiology techniques to (1) define the catchment area of $\mathrm{BDH},(2)$ identify clustering of TB in the BDH catchment area and (3) investigate factors associated with high rates of TB. The approach aimed to illustrate the local TB burden in the context of the geography of this remote region of PNG, using mapping to illustrate the results as a complement to the underlying quantitative spatial analysis.

\section{STUDY POPULATION AND METHODS}

\section{Study setting and patient cohort}

Patient data were obtained from the BDH TB patient register, which includes all patients diagnosed and commenced on TB treatment at $\mathrm{BDH}$, as described previ- 
ously. ${ }^{2}$ TB patients may be bacteriologically confirmed using smear microscopy or diagnosed clinically as occurs for the majority of cases in the Balimo region, in accordance with the World Health Organization (WHO) case definitions and PNG National Tuberculosis Management Protocol. ${ }^{2-4}$ In this study, patient locations were identified as the first village recorded as a residential address for each patient. Out of 1614 TB patients registered from 26 April 2013 to 25 February/2017, 1568 were mapped after excluding patients from outside Western Province $(n=13)$ and those for whom a residential address could not be determined $(n=33)$.

\section{Geographic and population data}

This study focused on the Balimo Urban (population 4394), Gogodala Rural (population 33 033) and Bamu Rural (population 13 432) LLG areas. In PNG, LLG areas are subdivided into rural wards and urban areas and further subdivided into census units. For this study, each patient's location was matched to a census unit and from there to an electoral ward based primarily on PNG census data or, alternatively, on the 2012 and 2017 PNG government election polling schedules. ${ }^{5,6}$ Instances of alternate local names were checked and confirmed locally.

Provincial, district and LLG boundary data and latitude and longitude coordinates of census units were obtained from the PNG National Statistical Office and census data. For coordinates that could not be obtained from census data, alternate sources including ArcGIS Online (Esri, Redlands, CA, USA) and a 2018 Google search were used. Population data for electoral wards used the 2011 national census figures ${ }^{7}$ to describe the underlying population at risk in the cluster analyses and logistic regression. Population size was not projected to later years as ward-level population growth data were not available.

\section{Mapping and cluster analyses}

The residential locations of TB patients diagnosed at $\mathrm{BDH}$ were mapped to identify the $\mathrm{BDH}$ catchment area (i.e. the region served by the hospital as defined by the origins of TB patients who have travelled to the hospital). Mapping of residential locations was primarily based on census unit-level coordinates. However, patients from some locations were mapped based on the average coordinates of a combination of census units as the precise census unit was rarely known for these patients. Average ward coordinates were calculated using the Geographic Midpoint Calculator available in 2017. There were four locations where all census units within a ward were averaged and four locations where the averaged coordinates included several census units within a ward. Towns and villages are depicted in Figures 1 to 3 spatially as dots as we did not have access to georeferenced boundaries at the ward level for this region of PNG.

Cluster analyses were undertaken separately for the Gogodala and Bamu regions using averaged wardlevel coordinates and ward-level population data. The Gogodala region included the 39 Gogodala Rural LLG wards plus Balimo Urban LLG; the Bamu region included the 19 Bamu Rural LLG wards. ${ }^{7}$ Eleven patients located within Western Province but outside the Gogodala and Bamu regions were excluded from the cluster analyses.

Cluster analyses based on paediatric and extrapulmonary TB cases were undertaken to compare clusters in these patient groups to the overall cluster analysis. These subanalyses used the same underlying population and coordinate data but with case data restricted to patient subgroups in the Gogodala region only. Age-stratified population data were not available for the wards in this region, so geographic differences in age distribution were not taken into account in the overall analysis.

The spatial scan statistic was calculated using $\mathrm{SaTS} \mathrm{Can}^{\mathrm{TM}}$ (version 9.6) (SaTScan, Boston, MA., USA). ${ }^{8}$

A discrete Poisson probability model was used because occurrence of the disease is rare. ${ }^{9,10}$ The data were scanned for areas with either high- or low-rate clusters. A circular spatial window was used, and the maximum spatial cluster size was set at the default size of $50 \%$ of the population at risk. The analyses were run with the default 999 replications with statistical significance set at $P<0.05$. Secondary clusters that were significant were non-overlapping Gini clusters. These clusters are selected to maximize the Gini index, which is a measure of statistical dispersion, and which can provide evidence of the best non-overlapping clusters to report from one larger cluster or multiple smaller clusters. ${ }^{10,11}$ Shapefiles depicting cluster areas were generated using SaTScan ${ }^{\mathrm{TM}}$. All maps were created using ArcGIS ArcMap 10.4.1(Esri, Redlands, CA., USA) and used the World Topographic Map basemap layer provided within the ArcGIS Online package. 


\section{Investigation of high-rate TB clusters}

For wards in the Gogodala region, univariate and multivariate logistic regression were used to investigate the relationship between ward-level demographic and geographic variables and the occurrence of wards in significant high-rate TB cluster areas. Based on ward-level population data, the predictor variables included gender ratio (total males/total females), housing density (total ward population/total number of households in the ward) and distance from Balimo (distance in kilometres from the averaged Balimo coordinates to the averaged ward coordinates). Distance was calculated using the National Hurricane Center Latitude/Longitude Distance Calculator. $^{12}$ Statistical analyses were performed using Stata/IC version 14 (StataCorp LLC, College Station, TX., USA).

\section{ETHICS APPROVAL}

This study received local approval from the Middle Fly District Health Service and the Evangelical Church of PNG Health Service. Human research ethics approval was received from the James Cook University Human Research Ethics Committee (H6432) and the PNG Medical Research Advisory Committee (MRAC No. 17.02).

\section{RESULTS}

The 1568 TB patients were identified at 90 localities across Western Province. These locations, shown in Fig. 1 , are based on census unit-level coordinates (averaged where relevant; see methods) and delineated by the LLG boundaries of Western Province. The catchment area is depicted with the majority of patients originating from the Balimo Urban $(n=252)$ and Gogodala Rural $(n=1010)$ LLG areas with a large number also in Bamu Rural LLG $(n=295)$. Eleven patients were located in other LLGs in Western Province.

\section{Cluster analyses}

High- and low-rate TB clusters are described in Table 1, and depictions in Fig. 2 (for the Gogodala region, $n=1262$ ) and Fig. 3 (for the Bamu region, $n=295$ ) are based on the ward-level population and TB patient data. Cluster numbers included in Table 1 correspond to the cluster numbers depicted in Fig. 2 and 3. The optimal Gini coefficients were found at $20 \%$ of the population in the Gogodala region and at $10-12 \%$ of the population in the Bamu region; paediatric TB and extrapulmonary TB subgroups were at $12 \%$ and $20 \%$, respectively. Only clusters with less than these proportions of the population at risk were reported for each region. In the Gogodala region, high-rate clusters were generally identified closer to Balimo, while low-rate clusters were seen on the outskirts of the region (Fig. 2). This trend continued to be evident for the paediatric $(n=283)$ and extrapulmonary TB ( $n=978$ ) subgroups (Fig. 2$)$. In the Bamu region, three high-rate clusters were identified in the lower regions of the Bamu and Gama Rivers; low-rate clusters were identified further along the Gama River and in the far north of the Bamu Rural LLG (Fig. 3).

The logistic regression results for predictors of wardlevel high-rate TB clusters are summarized in Table 2. In both the univariate and multivariate analyses, wards in high-rate TB clusters were associated with closer proximity to Balimo. Housing density had an odds ratio of 0.63 (95\% Cl: 0.34-1.20) in the univariate analysis, while the odds ratio in the multivariate analysis was $1.26(95 \% \mathrm{Cl}$ : $0.55-2.90$ ), suggesting confounding between housing density and distance from Balimo in this analysis.

\section{DISCUSSION}

This study examined the spatial distribution of TB patients diagnosed at BDH. The extensive hospital catchment area highlights the considerable distance that people travel to seek care for TB symptoms; however, the capacity to travel may help define and explain the lower case numbers in communities located further away from a health centre. In the Gogodala and Bamu regions, both high- and low-rate TB clusters were identified, illustrating the heterogeneity of reported TB burden across the region with a substantially higher TB burden evident in closer proximity to Balimo.

Most villages in the Gogodala Rural LLG had TB cases identified during the study period. Villages with no reported TB patients were predominantly located south of Balimo near the Fly River. Geographic challenges may be particularly important for people from this area as travel to either Balimo or Daru is lengthy, and fuel to travel by motorized boat to Daru is expensive. However, some patients were reported from the Gogodala region between Balimo and the Fly River, which may reflect a choice to travel to Balimo or, potentially, referral from a peripheral health facility in the region. Overall, villages 
Fig. 1. Map of Western Province, Papua New Guinea, with orange dots representing towns and villages (at the census unit level) where tuberculosis patients were identified in this study, differentiated by local level government area.

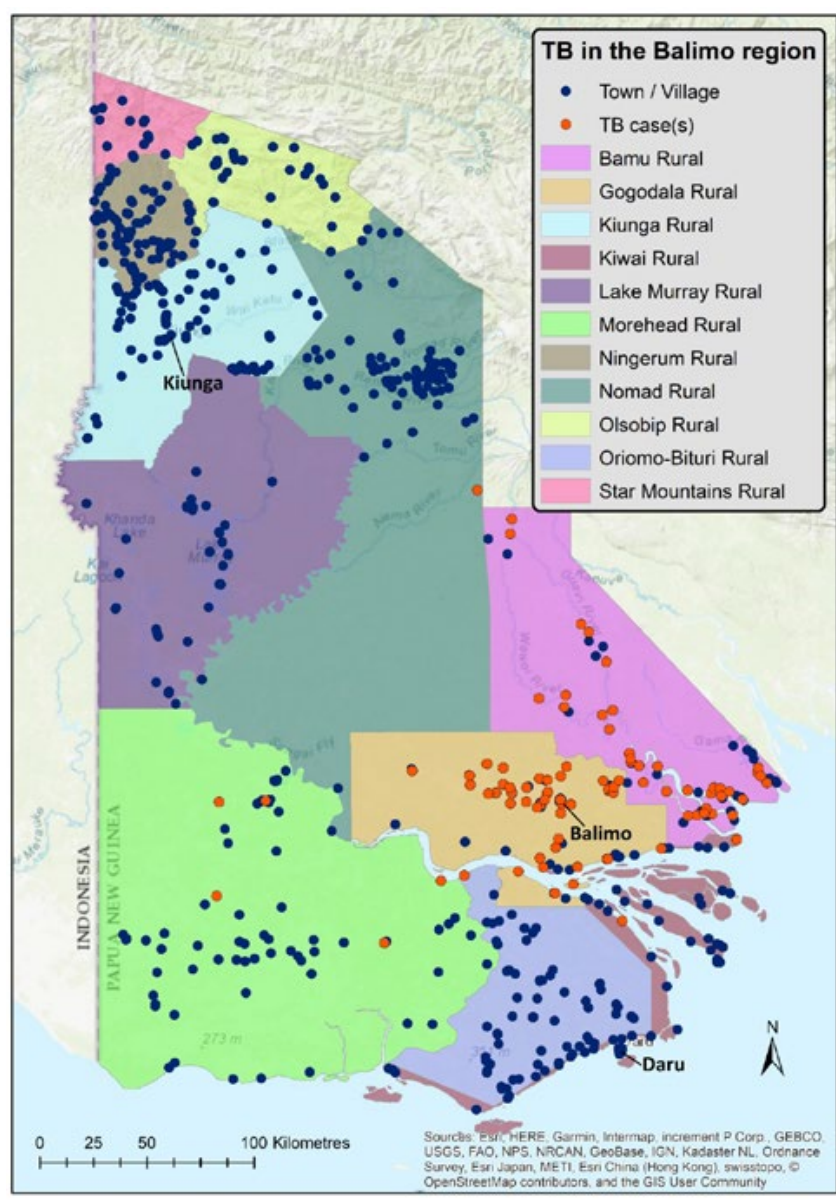

Map of Western Province, Papua New Guinea, with orange dots representing towns and villages (at the census unit level) where tuberculosis patients were identified in this study, differentiated by local level government area.

with no or low rates of TB should be noted for future investigation to identify people symptomatic for TB and describe treatment-seeking practices.

In this study, low-rate clusters occurred in more remote areas, while closer proximity to Balimo was a predictor of a ward located in a high-rate TB cluster. This association is potentially linked with underdiagnosis of TB in more remote areas as less arduous travel will promote better access to care. If villages in high-rate TB clusters reflect accurate rates of TB for the region more generally, villages with low rates of TB may indicate underdiagnosis of TB and are sites where active TB investigations should be undertaken. This finding is important as other research from our group has described potential underdiagnosis of TB in the Balimo region. ${ }^{13}$
Table 1. High- and low-rate tuberculosis clusters identified in wards in the Gogodala and Bamu regions of Western Province, Papua New Guinea using the spatial scan statistic

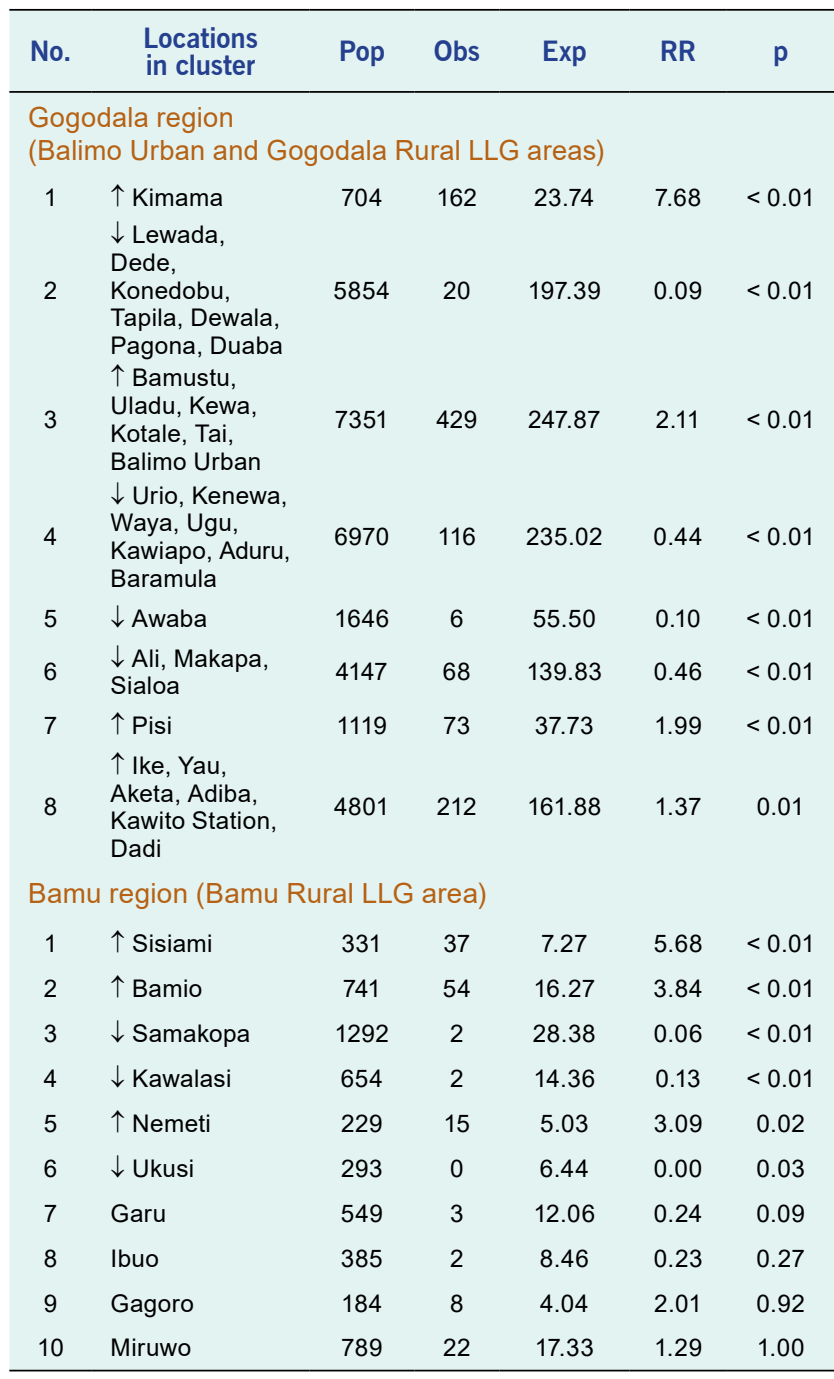

LLG: local level government; Pop: population; Obs: observed cases; Exp: expected cases; RR: relative risk; TB: tuberculosis

Statistical significance was set at $P<0.05$. High- and low-rate clusters that were significant are denoted with up- and down-arrows, respectively. The number of observed cases of TB are all those diagnosed for the cluster locations over the study period (26 April 2013 - 25 February 2017)

Table 2. Univariate and multivariate logistic regression examining ward-level predictors of high-rate TB clustering in the Gogodala region

\begin{tabular}{|c|c|c|c|c|c|}
\hline \multirow[b]{2}{*}{$\begin{array}{l}\text { Predictor } \\
\text { variables }\end{array}$} & \multicolumn{3}{|c|}{ Univariate } & \multicolumn{2}{|c|}{ Multivariate } \\
\hline & $\mathbf{n}$ & OR $(95 \% \mathrm{Cl})$ & p & $\begin{array}{c}\text { OR } \\
(95 \% \mathrm{Cl})\end{array}$ & p \\
\hline Gender ratio* & 40 & $\begin{array}{c}0.77 \\
(0.00-242.74)\end{array}$ & 0.93 & - & - \\
\hline $\begin{array}{l}\text { Housing } \\
\text { density }^{\dagger}\end{array}$ & 40 & $\begin{array}{c}0.63 \\
(0.34-1.20)\end{array}$ & 0.14 & $\begin{array}{c}1.26 \\
(0.55- \\
2.90)\end{array}$ & 0.58 \\
\hline $\begin{array}{l}\text { Distance from } \\
\text { the town of } \\
\text { Balimo }^{\ddagger}\end{array}$ & 40 & $\begin{array}{c}0.87 \\
(0.79-0.95)\end{array}$ & $<0.01$ & $\begin{array}{c}0.86 \\
(0.77- \\
0.96)\end{array}$ & $<0.01$ \\
\hline
\end{tabular}

$\mathrm{Cl}$ : confidence interval; OR: odds ratio

*Gender ratio is defined as total males/total females.

tHousing density is defined as number of persons/number of households.

tDistance from the town of Balimo is based on distance between average ward coordinates in kilometres. 
Fig. 2. Geographic distribution of high-rate (red circles) and low-rate (green circles) tuberculosis clusters identified in the analysis of wards in the Gogodala region in Western Province, Papua New Guinea. Cluster analyses are depicted for (a) all patients, (b) paediatric TB patients and (c) extrapulmonary TB patients.

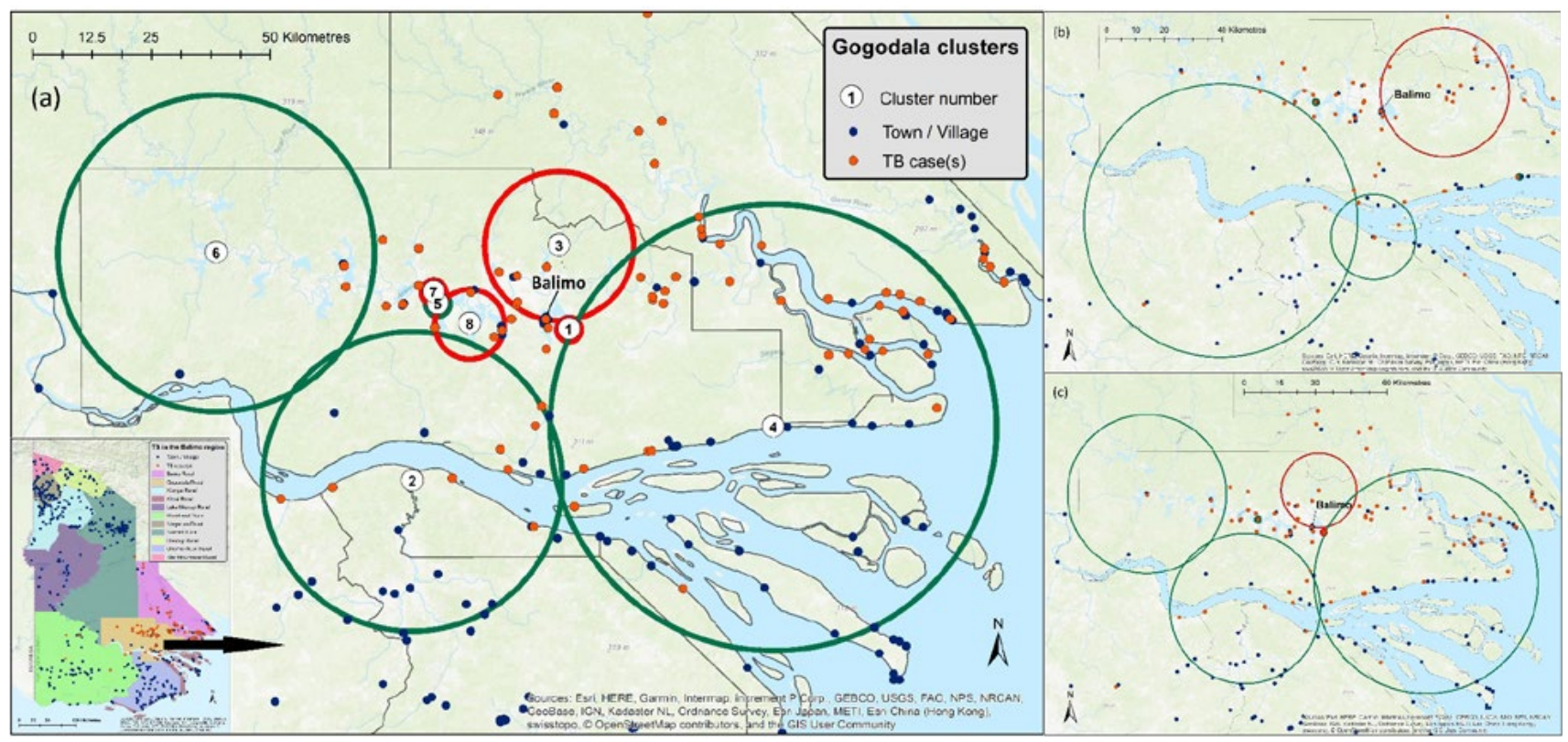

Orange dots represent locations where TB patients were identified and may include multiple TB patients. (Map sources: Esri, HERE, Garmin, Intermap, increment $P$ Corp., GEBCO, USGS, FAO, NPS, NRCAN, GeoBase, IGN, Kadaster NL, Ordnance Survey, Esri Japan, METI, Esri China (Hong Kong Special Administrative Region), swisstopo, (C) OpenStreetMap contributors and the GIS User Community)

Fig 3. Geographic distribution of high-rate (red circles) and low-rate (green circles) tuberculosis clusters identified in the analysis of wards in the Bamu Rural local level government area in Western Province, Papua New Guinea.

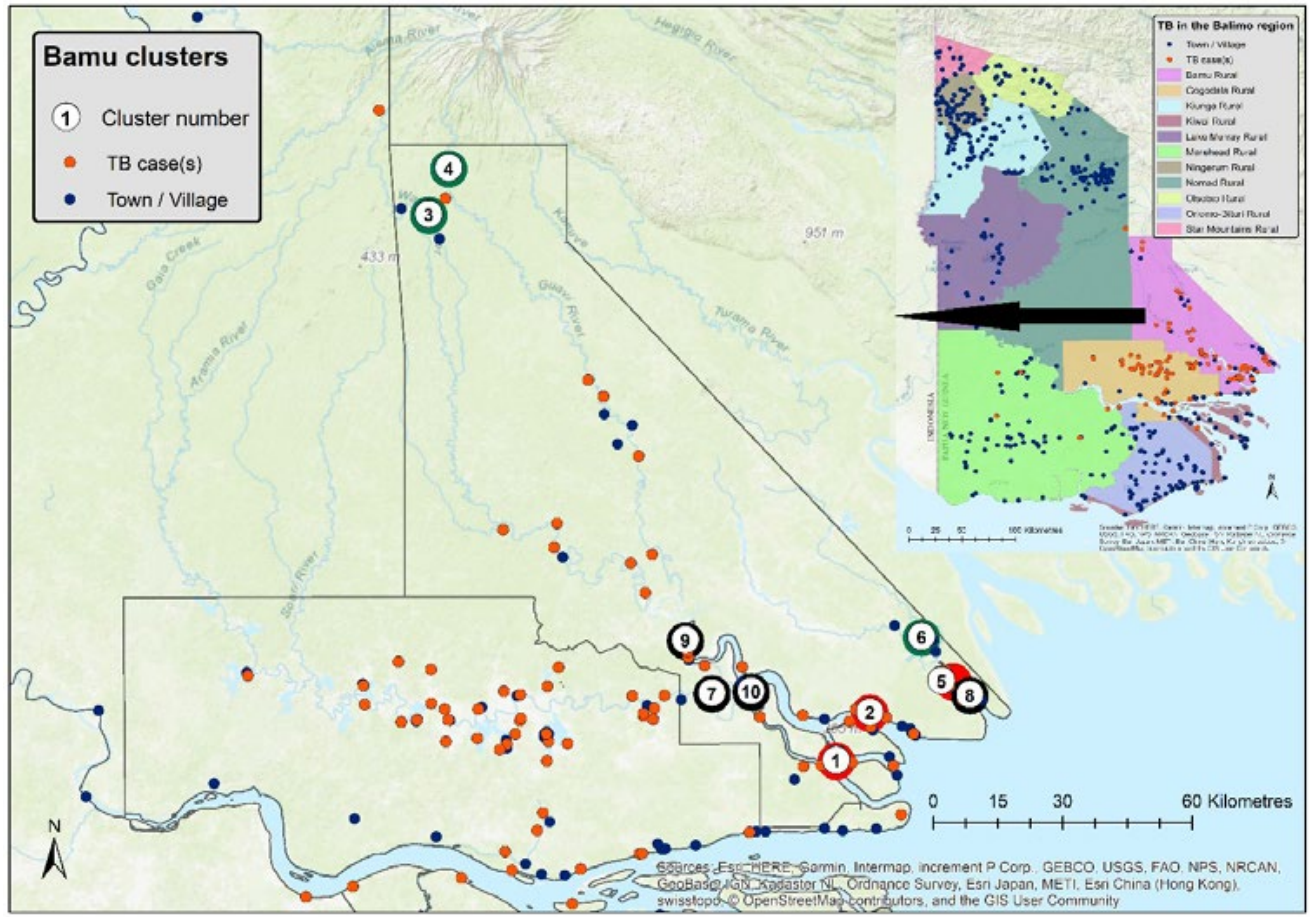

Clusters that were not statistically significant are shown as black circles. Orange dots represent locations where TB patients were identified and may include multiple TB patients. (Map sources: Esri, HERE, Garmin, Intermap, increment P Corp., GEBCO, USGS, FAO, NPS, NRCAN, GeoBase, IGN, Kadaster NL, Ordnance Survey, Esri Japan, METI, Esri China (Hong Kong Specia Administrative Region), swisstopo, (c) OpenStreetMap contributors and the GIS User Community) 
The possibility of underdiagnosis of TB was emphasized by results from the cluster analysis for the Bamu Rural LLG region. The high-rate clusters in three of these wards are the result of non-routine active case-finding activities. During an eight-day period in March 2016, 96 patients from eight villages in the Bamu Rural LLG region, including villages in these three high-rate cluster wards, were diagnosed with TB. By comparison, only 31 patients from these eight villages were diagnosed over the remainder of the study period. These diagnoses demonstrate the potential of an even higher burden of TB in remote and difficult-to-reach locations, reflecting people who may not otherwise have been diagnosed with TB. The geographically distant low-rate clusters seen in the Bamu Rural LLG likely reflect a combination of access challenges and the possibility of travel by patients to health centres other than BDH for TB care.

Other studies have described higher TB density in regions with closer proximity to urbanized areas and delayed treatment-seeking in people who travelled to a health facility by foot, while increased distance and poorer access to health facilities have been associated with diagnostic delay in some resource-limited settings. ${ }^{14-17}$ In addition, urbanization has been associated with higher rates of TB as a result of factors such as overcrowding and increased TB transmission risk; ${ }^{18,19}$ however, it is notable that housing density in Balimo was below average for the 40 Gogodala region wards (density of 6.9 people per household compared to an average of 7.4). Previous research in PNG has noted the importance of challenging travel in the context of TB care, including in the Gogodala region where travel is primarily by boat or by foot. ${ }^{20,21}$ In addition, where travel by boat is possible, socioeconomic factors and affordability of fuel will play a role in the ability to travel. ${ }^{20}$ Other factors, including proximity to a health facility, health worker training and local TB awareness activities have been associated with increased TB notifications. ${ }^{22-25}$ In the Gogodala region, possible reasons for locations with high case density include the presence of an actively staffed aid post or health clinic that regularly refers presumptive TB patients or increased case-finding or awareness activities.

High rates of paediatric and extrapulmonary TB have previously been identified in the Balimo region. ${ }^{2}$ Separate cluster analysis of these patient subgroups identified similar patterns to the overall distribution of TB in the Gogodala region. This finding may indicate similar TB transmission patterns across the region as well as consistency in the approach to identifying TB in a region where diagnoses are predominantly based on clinical signs and symptoms.

Awaba has the largest health centre in the Gogodala region outside of Balimo. The low-rate cluster identified in the Awaba ward is due to registration of TB patients diagnosed and started on TB treatment at the Awaba Health Centre instead of at BDH. The Awaba TB register was not available for this study, although TB incidence at the centre was estimated to be 381 cases per 100000 people per year in a 2011 Western Province TB evaluation study. ${ }^{26}$

In our study, the 11 patients from within Western Province but outside the Gogodala and Bamu regions may be important when considering importation of TB into the Gogodala region. Seven of these patients had alternative addresses recorded within the Gogodala and Bamu regions, including two at logging camps and one at a school. This suggests mobility of people in the region, particularly in the context of education and employment, which is important when considering that schools and workplaces can be important sites of TB transmission. ${ }^{27,28}$

In this analysis, it was assumed that a TB patient's first recorded address was where they were living at the time of registration. However, people with more than one address recorded may be more mobile, particularly if travelling between their residential and home villages (i.e. place of birth or family village) or workplace. Thus, some patient locations may not have reflected the location where TB infection occurred. An unknown number of TB patients were registered at smaller health facilities in the Balimo region. Although such patient numbers are likely to be low, these facilities will have influenced the analyses to an unknown extent. In addition, the TB patients identified and described here will not include Balimo-region patients diagnosed and commenced on treatment in the provincial capital of Daru. Finally, this analysis was based on population data collected in the 2011 PNG census. Thus TB rates may have been inaccurate for wards that experienced unusually high or low growth in the time before and during our study period of 2013 to 2017. 


\section{CONCLUSIONS}

This analysis provides insight into TB distribution in the $\mathrm{BDH}$ catchment area. The results provide baseline data about TB distribution across the region as well as targeted information that points to the need for villageand ward-specific TB investigations. In this region, TB clustering likely reflects the ease with which people can travel and seek treatment, demonstrating the importance of access to health services. However, investigation of high-rate TB clusters, as well as diagnoses resulting from targeted case-finding activities, emphasize the high potential for missed TB diagnoses in the region. The potentially substantial burden of undiagnosed TB in the extensive catchment area of $\mathrm{BDH}$ indicates an urgent need for active case-finding activities both to reduce TB disease burden and prevent ongoing transmission of TB in the region. This study may help focus a more targeted active TB case detection programme. Furthermore, these results emphasize the importance of targeted investment in resources and facilities in the Middle Fly District to improve and strengthen the provision of TB care in Western Province.

\section{Acknowledgements}

We thank Mr Suli Gayani and Mr Kimsy Waiwa for their support of this project and acknowledge Dr Patricia Graves and Dr Peter Wood for their contributions to the methods and design of this work. Research undertaken by Tanya Diefenbach-Elstob was supported by an Australian Government Research Training Program (RTP) Scholarship.

\section{References}

1. Aia P, Wangchuk L, Morishita F, Kisomb J, Yasi R, Kal M, Islam T. Epidemiology of tuberculosis in Papua New Guinea: analysis of case notification and treatment-outcome data, 2008-2016. West Pac Surveill Response J. 2018 Jun 15;9(2):9-19. doi:10.5365/wpsar.2018.9.1.006 pmid:30057853

2. Diefenbach-Elstob T, Graves P, Dowi R, Gula B, Plummer D, McBryde E, Pelowa D, Siba P, Pomat W, Warner J. The epidemiology of tuberculosis in the rural Balimo region of Papua New Guinea. Trop Med Int Health. 2018 Sep;23(9):1022-32. doi:10.1111/ tmi.13118 pmid:29920858

3. Department of Health. Papua New Guinea: national tuberculosis management protocol. Port Moresby, Papua New Guinea: Department of Health; 2011. Available from: https://www.adi.org.au/ wp-content/uploads/2016/11/National-Tuberculosis-ManagementProtocol-PNG-2011.pdf
4. World Health Organization. Definitions and reporting framework for tuberculosis - 2013 revision (updated December 2014). Geneva (Switzerland): World Health Organization; 2013. Available from: https:// apps.who.int/iris/bitstream/10665/79199/1/9789241505345 eng.pdf

5. Papua New Guinea Electoral Commission. Election commencing 23 June 2012: polling schedule for Middle Fly electorate. 2012 Available from: https://garamut.files.wordpress.com/2012/06/western_middle-fly-schedule.pdf

6. Papua New Guinea Electoral Commission. Election commencing 20th April 2017: polling schedule for Middle Fly Open electorate. Port Moresby: Papua New Guinea Electoral Commission. 2017. Available from: http://www.pngec.gov.pg/docs/default-source/default-document-library/polling-schedule-v6.pdf?sfvrsn=2

7. National Statistical Office. 2011 national population \& housing census: ward population profile - southern region. Port Moresby, National Statistical Office; 2014. Available from: http://www.nso.gov. pg/index.php/document-library?view $=$ download\&fileld $=64$

8. Kulldorff M, Information Management Services, Inc. SaTScanTM software for the spatial and space-time scan statistics. Boston, MA SaTScanTM; 2005. Available from: https://www.satscan.org/

9. Kulldorff M. A spatial scan statistic. Commun Stat Theory Methods. 1997;26(6):1481-96. doi:10.1080/03610929708831995

10. Kulldorff M. SaTScanTM user guide for version 9.6. Boston, MA: SaTScanTM; 2018. Available from: https://www.satscan.org/techdoc.html

11. Han J, Zhu L, Kulldorff M, Hostovich S, Stinchcomb DG, Tatalovich $Z$, Lewis DR, Feuer EJ. Using Gini coefficient to determining optimal cluster reporting sizes for spatial scan statistics. Int J Health Geogr. 2016 Aug 3;15(1):27. doi:10.1186/s12942-016-0056-6 pmid:27488416

12. National Hurricane Center. Latitude/longitude distance calculator. Miami, FL: US Dept of Commerce - National Oceanic and Atmospheric Administration. Available from: https://www.nhc.noaa.gov/ gccalc.shtml

13. Guernier V, Diefenbach-Elstob T, Pelowa D, Pollard S, Burgess G, McBryde ES, Warner J. Molecular diagnosis of suspected tuberculosis from archived smear slides from the Balimo region, Papua New Guinea. Int J Infect Dis. 2018 Feb;67:75-81. doi:10.1016/j. ijid.2017.12.004 pmid:29229499

14. Dangisso MH, Datiko DG, Lindtjørn B. Spatio-temporal analysis of smear-positive tuberculosis in the Sidama Zone, southern Ethiopia. PLoS One. 2015 Jun 1;10(6):e0126369. doi:10.1371/journal. pone.0126369 pmid:26030162

15. Yirgu R, Lemessa F, Hirpa S, Alemayehu A, Klinkenberg E. Determinants of delayed care seeking for TB suggestive symptoms in Seru district, Oromiya region, Ethiopia: a community based unmatched case-control study. BMC Infect Dis. 2017 Apr 20;17(1):292. doi:10.1186/s12879-017-2407-8 pmid:28427367

16. Babatunde OI, Bismark EC, Amaechi NE, Gabriel El, Olanike A-UR. Determinants of treatment delays among pulmonary tuberculosis patients in Enugu Metropolis, South-East, Nigeria. Health. 2015;7(11):1506-16. doi:10.4236/health.2015.711164

17. Demissie M, Lindtjørn B, Berhane Y. Patient and health service delay in the diagnosis of pulmonary tuberculosis in Ethiopia. BMC Public Health. 2002 Sep 25;2(1):23. doi:10.1186/1471-2458-2-23 pmid:12296975

18. Antunes JLF, Waldman EA. The impact of AIDS, immigration and housing overcrowding on tuberculosis deaths in São Paulo, Brazil, 1994-1998. Soc Sci Med. 2001 Apr;52(7):1071-80. doi:10.1016/ S0277-9536(00)00214-8 pmid:11266050 
19. Oppong JR, Mayer J, Oren E. The global health threat of African urban slums: the example of urban tuberculosis. African Geographical Review. 2015;34(2):182-95. doi:10.1080/19376812.2014.9 10815

20. Diefenbach-Elstob T, Plummer D, Dowi R, Wamagi S, Gula B, Siwaeya K, Pelowa D, Siba P, Warner J. The social determinants of tuberculosis treatment adherence in a remote region of Papua New Guinea. BMC Public Health. 2017 Jan 13;17(1):70. doi:10.1186/ s12889-016-3935-7 pmid:28086845

21. Ongugo K, Hall J, Attia J. Implementing tuberculosis control in Papua New Guinea: a clash of culture and science? J Community Health. 2011 Jun;36(3):423-30. doi:10.1007/s10900-010-93248 pmid:21161347

22. Datiko DG, Yassin MA, Theobald SJ, Blok L, Suvanand S, Creswell J, Cuevas LE. Health extension workers improve tuberculosis case finding and treatment outcome in Ethiopia: a large-scale implementation study. BMJ Glob Health. 2017 Nov 2;2(4):e000390. doi:10.1136/bmjgh-2017-000390 pmid:29209537

23. Dangisso MH, Datiko DG, Lindtjørn B. Accessibility to tuberculosis control services and tuberculosis programme performance in southern Ethiopia. Glob Health Action. 2015 Nov 20;8(1):29443. doi:10.3402/gha.v8.29443 pmid:26593274
24. Parija D, Patra TK, Kumar AMV, Swain BK, Satyanarayana S, Sreenivas A, Chadha VK, Moonan PK, Oeltmann JE. Impact of awareness drives and community-based active tuberculosis case finding in Odisha, India. Int J Tuberc Lung Dis. 2014 Sep;18(9):1105-7. doi:10.5588/ijtld.13.0918 pmid:25189560

25. Yassin MA, Datiko DG, Tulloch O, Markos P, Aschalew M, Shargie $\mathrm{EB}$, et al. Innovative community-based approaches doubled tuberculosis case notification and improve treatment outcome in Southern Ethiopia. PLoS One. 2013 May 27;8(5):e63174. doi:10.1371/ journal.pone.0063174 pmid:23723975

26. McBryde E. Evaluation of risks of tuberculosis in Western Province Papua New Guinea. Barton. Department of Foreign Affairs and Trade; 2012. Available from: https://www.burnet.edu.au/system/ publication/file/3606/2012_Evaluation_of_Risks_of_Tuberculosis _ in_Western_Province_PNG.pdf

27. Andrews JR, Morrow C, Walensky RP, Wood R. Integrating social contact and environmental data in evaluating tuberculosis transmission in a South African township. J Infect Dis. 2014 Aug 15;210(4):597603. doi:10.1093/infdis/jiu138 pmid:24610874

28. McElnay C, Thornley C, Armstrong R. A community and workplace outbreak of tuberculosis in Hawke's Bay in 2002. N Z Med J. 2004 Aug 20;117(1200):U1019. pmid:15475989 